\title{
Are there critical bands in kinesthesia?
}

\author{
L. A. JONES and I. W. HUNTER \\ Massachusetts Institute of Technology, Cambridge, Massachusetts \\ and \\ R. J. IRWIN \\ University of Auckland, Auckland, New Zealand
}

\begin{abstract}
The effect of changing the bandwidth of noise on the ability of human subjects to detect a $10-\mathrm{Hz}$ sinusoidal movement signal was measured in two experiments. The objective of these studies was to investigate whether critical bands exist for the kinesthetic system, as has been demonstrated for the auditory and tactile systems. It was found that subjects' ability to detect a $10-\mathrm{Hz}$ sinusoidal movement stimulus embedded in noise was not influenced by the bandwidth of the noise over a range of 4-10 Hz. These findings suggest that, if a critical filter does exist for this system, it would have to be greater than $10 \mathrm{~Hz}$.
\end{abstract}

The ability to detect a limb movement depends on a number of factors, including the particular joint moved, whether or not the muscles are contracting during the limb movement, and the velocity of the movement. The threshold for detecting movements imposed on more proximal joints, such as the shoulder or hip, is lower than that for more distal joints, such as those in the fingers or toes (Hall \& McCloskey, 1983; Refshauge, Chan, Taylor, \& McCloskey, 1995). This finding is not surprising, given that proximal joints move more slowly than distal joints and that rotation of proximal joints results in larger displacements of the end-point of the limb-namely, the fingertip or the toes-than does the same angular rotation at a more distal joint. If thresholds are analyzed in terms of displacement of the end point of the movement and not in terms of joint rotation, distal joints are superior to proximal joints (Hall \& McCloskey, 1983).

If the muscles acting on a joint are actively contracting when a movement is imposed, the thresholds for detecting the movement can be up to 10 times smaller than those measured at the same movement velocity when the muscle is relaxed (Fitzpatrick \& McCloskey, 1994; Taylor \& McCloskey, 1992). This effect is most evident at lower movement velocities, which are associated with higher thresholds, and presumably reflects the increased sensitivity of muscle spindle receptors to the movement. For the elbow joint, it has been found that the effect of muscle activation on movement detection thresholds is the same independently of the amplitude of force $(5$ or $20 \mathrm{~N})$ exerted (Taylor \& McCloskey, 1992).

The velocity of a movement imposed on a joint also influences the ability of the subject to detect the movement.

This research was supported by the Institute for Robotics and Intelligent Systems (IRIS), a Canadian Network of Centres of Excellence. Correspondence concerning this article should be addressed to L. A. Jones, Department of Mechanical Engineering, Room 3-148, Massachusetts Institute of Technology, 77 Massachusetts Ave., Cambridge, MA 02139 (e-mail: ljones@mit.edu).
For the distal interphalangeal joint of the middle finger, the threshold for detecting the movement decreases from approximately $8 \mathrm{deg}$ at $1.25 \mathrm{deg} / \mathrm{sec}$ to $1 \mathrm{deg}$ at a velocity of $10 \mathrm{deg} / \mathrm{sec}$. The threshold then remains constant at $1 \mathrm{deg}$ over a velocity range of 10-80 deg/ $\mathrm{sec}$ (Gandevia, Hall, \& McCloskey, 1983). The velocity region of optimal sensitivity is the one that subjects use spontaneously when they are asked to make voluntary pointing movements involving this joint (Hall \& McCloskey, 1983). The thresholds described above were obtained while unidirectional movements were imposed on the joint. When sinusoidal movements are imposed on the joint, thresholds for detecting movements are lower, and, just as increased velocity aids in the detection of unidirectional movements, thresholds decrease with increasing frequency (Goodwin, 1976; Kokmen, Bossemeyer, \& Williams, 1977). For the proximal interphalangeal joint of the index finger, the threshold decreases from approximately $2 \mathrm{deg}$ at $0.1 \mathrm{~Hz}$ to $0.16 \mathrm{deg}$ at $10 \mathrm{~Hz}$ (Goodwin, 1976).

The focus of many studies of human movement perception has been on the measurement of absolute thresholds and how they change as a function of the velocity of the movement and of the joint being moved. Differential thresholds have been measured in one study in which Gaussian white-noise displacement perturbations were applied simultaneously to the arms (Jones, Hunter, \& Irwin, 1992). The subjects were required to indicate which of the two low-pass filtered (with a cutoff at $15 \mathrm{~Hz}$ ) signals had the larger amplitude (defined in terms of the root-mean square [RMS] deviation). It was found that subjects could reliably discriminate an $8 \%$ difference in the RMS deviation amplitude of two random displacements and, at the smallest amplitude, were able to resolve a $5-\mu \mathrm{m}$ difference between the two perturbations (Jones et al., 1992). The latter accomplishment probably reflects the perception of skin indentation and not movement of the forearm.

The results from the experiment described above may be stimulus limited-that is, they may be a function of using Gaussian noise waveforms. It is not known what 
effect changing the stimulus waveform or bandwidth has on the ability of human subjects to discriminate between movements. The present experiments were designed to determine the frequency sensitivity of the kinesthetic system by measuring the ability of subjects to detect sinusoidal movement signals embedded in noise of different bandwidths. For the auditory system, it has been shown that when a sinusoidal signal is masked by noise, only the frequencies in the noise that are close to the frequency of the tone influence the detectability of the tone. The range of frequencies in the noise that can mask the tone is known as the critical band.

The concept of the critical band arose from experiments in which the threshold for detecting a sinusoidal signal was measured as a function of the bandwidth of the masking noise stimulus that was centered at the signal frequency. Fletcher (1940) and Schooneveldt and Moore (1989) found that the threshold for detecting a tone increased initially as the noise bandwidth increased and then reached a plateau with further increases in the noise bandwidth. Fletcher hypothesized that these changes in signal threshold occurred because of filtering processes in the peripheral auditory system, which result in listeners' using an auditory filter with a center frequency close to that of the signal when they are attempting to detect a signal in a noise background. The filter passes the signal but removes much of the noise, and only those components of the noise passing through the filter are able to mask the signal. The threshold for detecting the signal is, therefore, determined by the amount of noise passing though the filter and by the bandwidth of the noise, provided it is less than the filter bandwidth (Moore, 1997). Once the noise bandwidth exceeds the filter bandwidth, further increases in the noise bandwidth will not influence the amount of noise passing through the filter, and the signal threshold will, therefore, remain constant. The bandwidth at which the threshold stops increasing is called the critical bandwidth.

For the tactile system, masking stimuli have been used to identify the different sensory channels used to process vibrotactile information, which, for the glabrous (nonhairy) skin, covers a frequency range of $0.4-500 \mathrm{~Hz}$ (Bolanowski, Gescheider, Verrillo, \& Checkosky, 1988). Just as auditory filters have been proposed to account for the effects of noise bandwidth on the detection of auditory stimuli, separate vibrotactile information-processing channels have been hypothesized to exist in the tactile system, each operating over a limited range of frequencies (Bolanowski et al., 1988; Gescheider, Verrillo, \& Pelli, 1992). It appears that there are four channels available for processing vibrotactile stimuli with overlapping frequency characteristics, and each channel is associated with a specific type of mechanoreceptor in the skin (Bolanowski et al., 1988). The four channels can also be differentiated on the basis of their sensitivity to changes in skin-surface temperature and whether they are capable of spatial and temporal summation.
It is assumed that the channel most sensitive to a vibrotactile stimulus mediates detection of the signal and that maskers are effective only inasmuch as they are processed by the channel used to detect the signal. Masking stimuli that activate one of these vibrotactile channels do not interfere with the detection of stimuli by other channels unless they are of an intensity that is sufficient to excite other channels as well (Gescheider, Verrillo, \& Van Doren, 1982; Makous, Friedman, \& Vierck, 1995). The channels that mediate the perception of low-frequency vibrotactile stimuli have been identified as the non-Pacinian (NP) I channel, which has a nominal operating range of $10-$ $100 \mathrm{~Hz}$, and the NP III channel, which operates over a frequency range of $0.4-100 \mathrm{~Hz}$. Neither channel is capable of spatial summation, and the NP III channel, unlike the NP I channel, is affected by skin-surface temperature (Bolanowski et al., 1988).

\section{EXPERIMENTS 1 AND 2}

Masking stimuli spanning different frequency ranges have not been used in studies of the kinesthetic system, and so it is not known what range of frequencies can influence the perception of movement and whether critical bands exist for this system. Critical bands are a feature of other sensory systems, and their analysis has provided important cues regarding the processing of information in these systems. In the kinesthetic system, there does not appear to be any strict partitioning of sensory input on the basis of frequency, although movement and position signals can be encoded independently (Clark, Burgess, Chapin, \& Lipscomb, 1985). The objective of the present experiments was to determine whether the detection of a $10-\mathrm{Hz}$ sinusoidal movement perturbation applied to the arms was affected by the bandwidth of the noise in which the signal was embedded. If, as expected, detection does change with the noise bandwidth, this would provide support for the concept of a critical band in kinesthesia. In the present experiments, the number of sinusoids making up the noise stimulus, which had a bandwidth of either 4 or $10 \mathrm{~Hz}$, was kept constant at 21 , so that, in both experiments, the noise would be perceived as being random by the observers. This means that the overall power remained the same in both experiments. The findings from the auditory system and the known properties of the kinesthetic system suggested that the critical band, if it existed, would be less than $10 \mathrm{~Hz}$. On the basis of that assumption, it was hypothesized that, as the noise bandwidth decreased from 10 to $4 \mathrm{~Hz}$, the threshold for detecting the signal should decrease. If the critical band exceeded $10 \mathrm{~Hz}$, however, the threshold would not be smaller for the $10-\mathrm{Hz}$ than for 4$\mathrm{Hz}$ band of noise.

\section{Method}

Subjects. Five normal healthy adult subjects ( 3 men and 2 women) participated in the two experiments. They included graduate students and research staff in the Department of Mechanical En- 


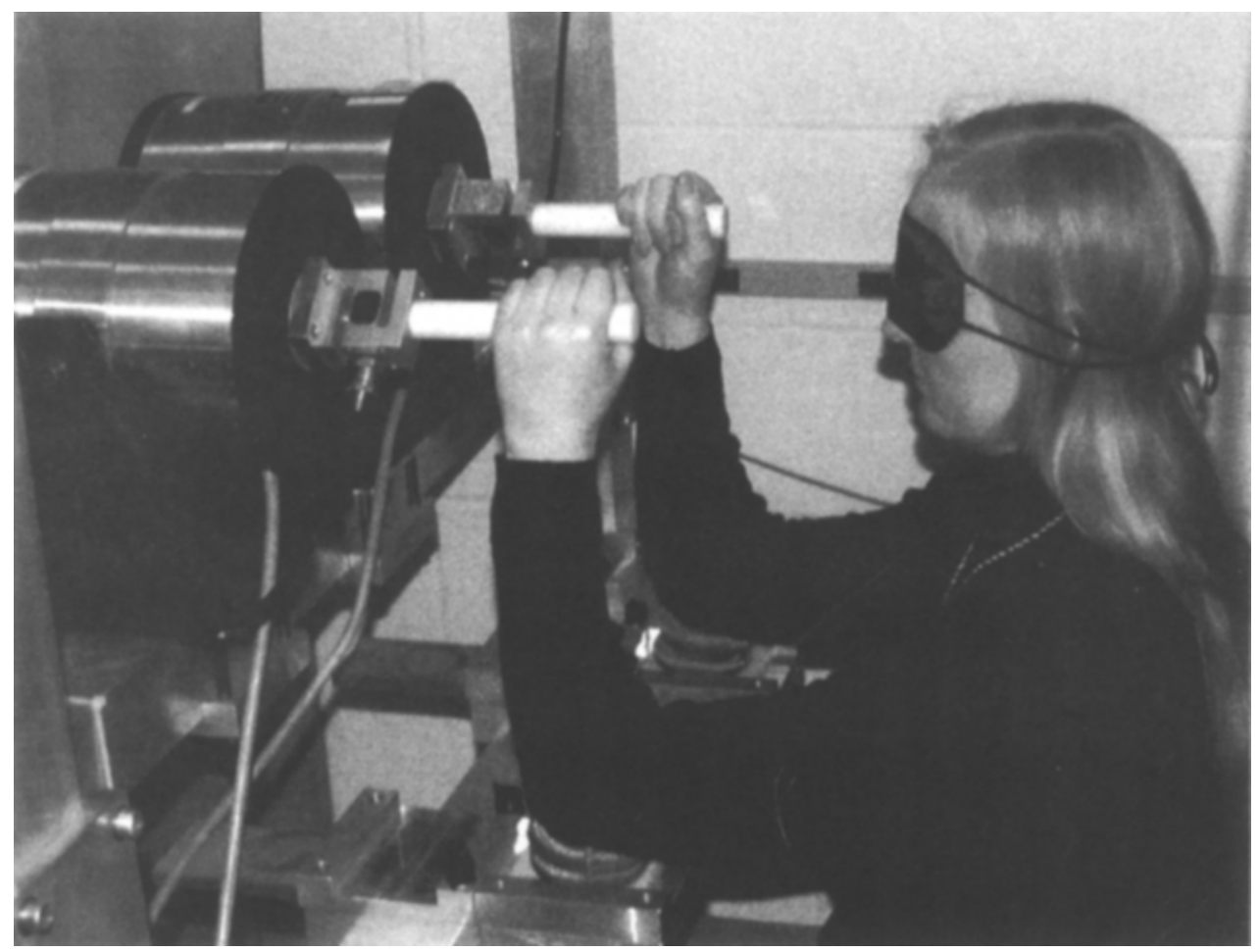

Figure 1. Photograph of a subject seated in the apparatus used in the experiment.

gineering at MIT. They had no known abnormalities of the neuromuscular system. They ranged in age from 24 to 41 years (mean, 30 years).

Apparatus. An experimental rig, measuring $1.2 \mathrm{~m}^{2}$ at the base and $2 \mathrm{~m}$ high, was constructed from square-bar aluminum struts. The apparatus is shown in Figure 1. Mounted in the center of the rig was a four-axis electrically adjustable seat, and in front of the chair was a horizontal beam on which elbow supports were positioned. Two powerful ( $\pm 440 \mathrm{~N}$ ) electromagnetic linear motors (Vibration Test Systems, Model VG 100-6) were bolted to the rig in front of the subjects, spaced $270 \mathrm{~mm}$ apart. Each motor was driven by a $500-\mathrm{W}$, class AB power amplifier (Techron, Model 5530) with a flat bandwidth from 0 to $10 \mathrm{kHz}$. The output noise levels of the amplifiers was $2 * 10^{-6}$ of the peak output. A 16-bit digital-to-analog converter (DAC; Tasco model TVXI) delivered voltage commands at a $1-\mathrm{kHz}$ rate to each power amplifier and, hence, to the actuator. Connected to the coil of each motor was a cylindrical rod that the subjects grasped firmly in their hands. Inductive position transducers (Data Instruments, Fastar model FS 380) that have a flat frequency response to more than $10 \mathrm{kHz}$ measured the displacements of the motors. The noise level of the position transducers, measured over a 0 to $10-\mathrm{kHz}$ bandwidth when the actuators, power amplifiers, and servosystem were on, was $4-\mu \mathrm{m}$ RMS deviation.

The motors delivered a displacement perturbation to each arm simultaneously for $5 \mathrm{sec}$. The perturbation delivered by the motors resulted in movements of the forearms as the subjects firmly grasped the rods projecting from the motors. At the largest amplitude presented, movement of the elbow joint and forearm would have averaged approximately 2-4 deg. In Experiment 1, the whitenoise stimulus was a $5-15 \mathrm{~Hz}$ band of noise ( $10-\mathrm{Hz}$ bandwidth) that had a flat spectrum and was corrected for the dynamics of the actuator and arm. It has been shown that, if more than seven sinusoids spread evenly over a frequency band with random phases are added together, they are perceived as being random (Sheridan \& Ferrell, 1974). In the present experiments, 21 sinusoids spread evenly across the bandwidth of $5-15 \mathrm{~Hz}(8-12 \mathrm{~Hz}$ in Experiment 2) were randomly assigned 21 phases that were equally spaced between $0^{\circ}$ and $360^{\circ}$ and then added together. The signal-plus-noise stimulus was the same noise with a $10-\mathrm{Hz}$ signal whose amplitude varied from 1 to 4.5 times the RMS deviation amplitude of the noise. In Experiment 2, the white-noise stimulus was an 8-12 $\mathrm{Hz}$ band of noise (bandwidth of $4 \mathrm{~Hz}$ ), and the signal amplitude again varied from 1 to 4.5 times the RMS deviation amplitude of the noise. The number of sinusoids was kept constant at 21 in the two experiments, and so the overall power remained the same. The power spectra of the stimuli used in each experiment are shown in Figure 2. The number of frequencies was kept constant at 21 so that the stimuli would appear to be random to the observer in both experiments. It was thought that, with a small number of frequencies, subjects may be able to detect periodicities in the stimuli. The relation between the relative amplitude of the signal (where 0 amplitude represents the noise stimulus) and the standard deviation of the motor displacement (without a subject connected to the apparatus) in the two experiments is shown in Figure 3. As can be seen in the figure, there is little difference between the standard deviations of the motor displacements in each amplitude condition in the two experiments.

Variations in the amplitude of the noise displacement can result from differences in the mechanical properties of the forearm, such as its stiffness and inertia. The standard deviations and variance of the noise and signal-plus-noise displacements were calculated on each trial, and the means of these two variables were computed for each amplitude condition. Subsequent analyses of these displacements were made, using the trials identified by the subjects as being hits. For any given subject, the displacement RMS deviation amplitude of the noise stimulus remained very constant across trials and, for different subjects, ranged from 0.2815 to $0.3119 \mathrm{~mm}$. 


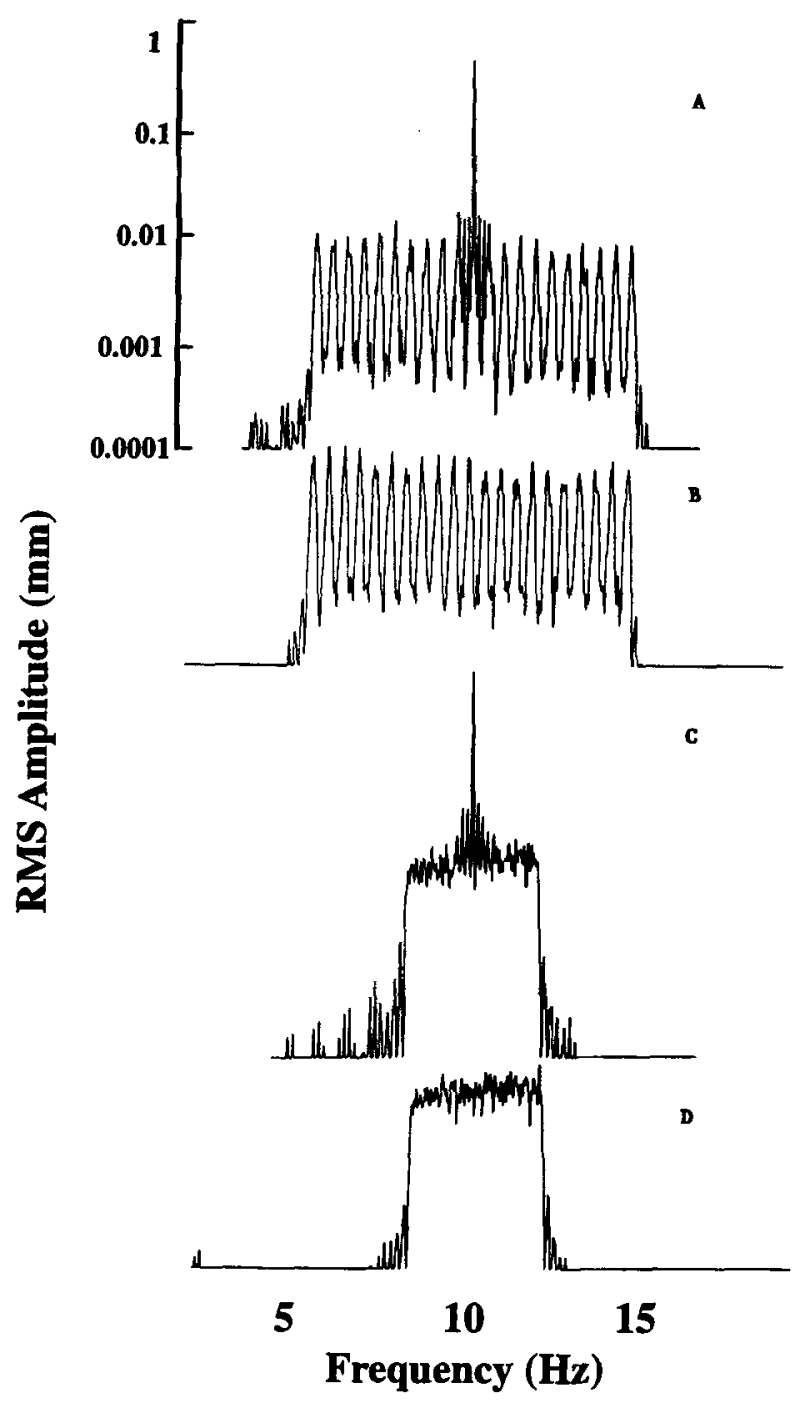

Figure 2. The power spectra of the stimuli used in the two experiments. $A$ is the signal-plus-noise stimulus, and $B$ is the noise stimulus used in Experiment 1 (5-15 Hz noise bandwidth). $C$ is the signal-plus-noise stimulus, and $D$ is the noise stimulus used in Experiment 2 (8-12 Hz noise bandwidth). The power spectra were calculated from 15 repetitions of the stimuli.

Displacement signals were recorded via 16-bit analog-to-digital converters (ADCs) at $100 \mathrm{~Hz}$ on each trial. The subject's response on each trial was also recorded. The ADCs and DACs formed part of a VXI instrumentation bus crate (Hewlett-Packard model E1401) connected to an IBM R6000/320 computer via VXI to microchannelbus MXI communication hardware (National Instruments model VXI-MC6000). The experimental procedures and servocontrols were all written in the $\mathrm{C}$ programming language.

Procedure. The subjects sat in the chair with each elbow joint resting in a support that was positioned so that the angle between the upper arm and the forearm was $90 \mathrm{deg}$. During the experiment, the subjects were blindfolded, to prevent them from using visual cues to facilitate the perception of movement, and they wore headphones to mask any auditory cues arising from the motors.
A forced-choice paradigm was used with trial-by-trial feedback of the correct answer. On each trial, a displacement perturbation was delivered simultaneously to each arm for $5 \mathrm{sec}$. The stimuli were presented in phase and simultaneously rather than successively, so that the subjects could make a direct comparison between the two stimuli, rather than rely on their memory of the first stimulus. There does not appear to be any evidence that suggests that kinesthetic stimuli presented to one arm mask the perception of stimuli presented to the contralateral arm.

The subjects were told that one of the two stimuli presented contained a $10-\mathrm{Hz}$ signal that had a larger amplitude than the other frequencies included in the stimulus and that they were to detect this signal. At the end of the perturbation, the subjects indicated within $20 \mathrm{sec}$ which displacement contained the signal by pulling the appropriate arm toward them. After a brief pause, the correct response (the arm that had experienced the signal + noise condition) was indicated to the subject by a $2-\mathrm{mm}, 100-\mathrm{msec}$ pulse delivered to the correct arm. The subjects had 10 practice trials prior to starting the experiment, to familiarize them with the experimental procedure and stimuli.

In both experiments, the amplitude of the $10-\mathrm{Hz}$ signal varied from 1 to 4.5 times the RMS deviation amplitude of the noise, and a total of eight different amplitudes (1, 1.5, 2, 2.5, 3, 3.5, 4, and 4.5) was presented in a random order, with 100 trials per amplitude. On each trial, it was randomly determined which motor (left or right) delivered the signal-plus-noise stimulus, and for each block of 100 trials, there was an equal number of signal-plus-noise stimuli presented by the left and right motors. To avoid fatigue, each subject was tested on 2 separate days, with 50 trials being presented at each of the eight amplitudes on both days. During both testing sessions, there was a pause (up to $3 \mathrm{~min}$ ) between each set of 50 trials. The experimental session typically lasted 50-60 min. Experiment 2 was conducted 6 months after Experiment 1.

The subjects were not given any explicit instructions regarding the cues to use to detect the signal-plus-noise stimulus and were simply told that the signal that they were attempting to identify had a larger amplitude than that of the background noise. They did not report any difficulties in performing the task and always made a response within $5 \mathrm{sec}$ of the termination of the movement perturbations.

\section{Results}

The relation between the relative amplitude of the signal and the percent correct is shown for each subject and both noise bandwidths in Figure 4. As was expected, as the amplitude of the signal increased, the subjects found it easier to detect, and, over the range investigated, there is a linear relation between the amplitude of the $10-\mathrm{Hz}$ signal and percent correct (Experiment 1, $r^{2}=.97$; Experiment 2, $r^{2}=.95$ ). There was virtually no difference between the arms in the percentage of correct responses (left arm, $49 \%$; right arm, $51 \%$ ).

For each subject, a least squares linear regression line with one free parameter (the intercept was set at $50 \%$ at 0 amplitude) was fitted to the relation between relative amplitude and percent correct, as is shown in Figure 4. A threshold was then calculated from the regression equations and was defined as the relative amplitude at the point of $71 \%$ correct responses. A threshold value of $71 \%$ correct was chosen in the present experiment in order to be consistent with earlier experiments on kinesthesia conducted in the laboratory, in which the transformed up- 


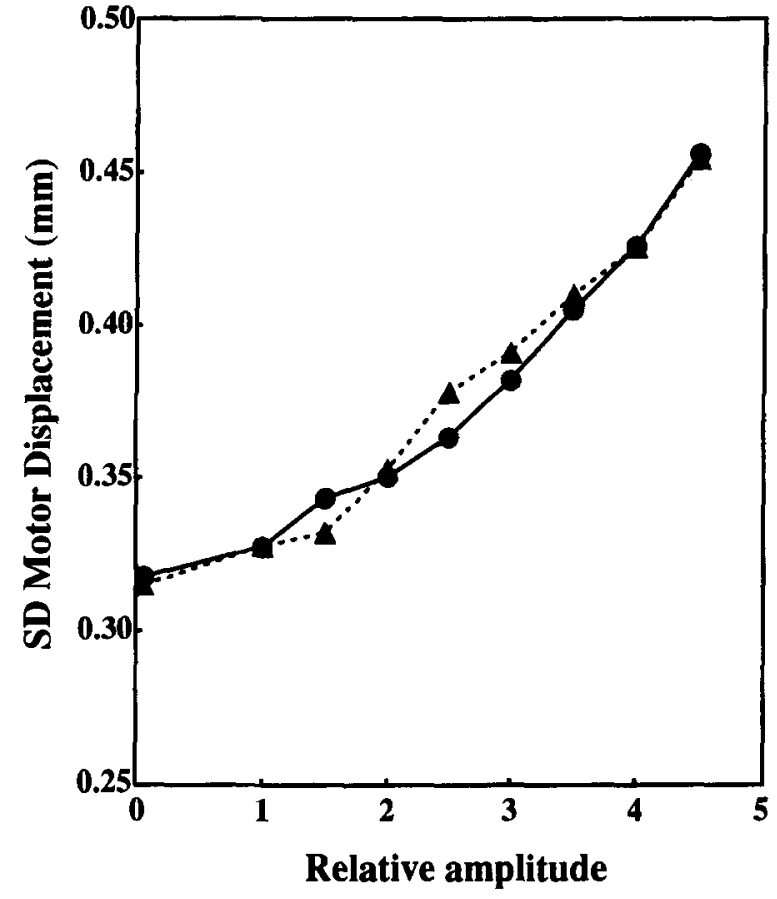

Figure 3. The mean of the standard deviations (SD) of the displacements of the motor recorded at each amplitude with the 10Hz bandwidth (circles and solid line) and the 4-Hz bandwidth of noise (triangles and dashed line). Ten measurements, each lasting $5 \mathrm{sec}$, were taken at each amplitude.

down method was used to calculate thresholds (see, e.g., Jones et al., 1992). As it was implemented, the latter procedure sought a stimulus level that corresponded to $71 \%$ correct performance.

In Experiment 1, with the $10-\mathrm{Hz}$ noise bandwidth the threshold was reached at a mean signal amplitude of 4.09 times the RMS deviation amplitude of the noise. For individual subjects, the thresholds ranged from 3.01 to 5.20 times the RMS deviation amplitude of the noise. In Experiment 2, with the 4- $\mathrm{Hz}$ noise bandwidth the mean threshold was achieved at 3.58 times the RMS deviation amplitude of the noise (range, 2.95-5.13). For 4 of the 5 subjects, the thresholds were higher in the first experiment with the $10-\mathrm{Hz}$ band of noise. An analysis of the thresholds measured in the two experiments revealed that there was no significant difference between the experiments $[t(4)=1.64, p=.09]$.

\section{Discussion}

The results of the present experiments indicate that the ability of subjects to detect a $10-\mathrm{Hz}$ movement signal was not significantly affected by decreasing the bandwidth of the noise in which the signal was embedded from 10 to $4 \mathrm{~Hz}$. The amplitudes of the components of the noise were kept constant when the bandwidth was changed, as was the case in the original auditory experiments on critical bands by Fletcher (1940). The number of sinusoids making up the noise stimulus was also kept constant in the present experiments, and so the overall power remained the same. The signal-to-noise ratio for detectability was, however, unaffected by the change in bandwidth, which would be expected if a filter was filtering out the noise around the signal. An examination of the individual subjects' data in Figure 4 does show, however, that the function for the $10-\mathrm{Hz}$ band falls below that for the $4-\mathrm{Hz}$ band of noise for 4 of the subjects tested, which suggests that there is a small decrease in the effectiveness of masking with noise bandwidth. This may well reflect chance variation in the data or, possibly, the experience gained through participating in Experiment 1 first. The latter is considered unlikely, because of the relatively long time interval between the two experiments (6 months).

If a critical band does exist for this system, which has not been demonstrated in the present experiments, it would have to be larger than $10 \mathrm{~Hz}$, and would not fall between 4 and $10 \mathrm{~Hz}$. The present results suggest that the critical band is not less than $10 \mathrm{~Hz}$, as the threshold did not decrease when the signal was embedded in the $4-\mathrm{Hz}$ noise bandwidth despite the more concentrated noise. If the critical band was less than $10 \mathrm{~Hz}$, the power in that band would have been reduced and so should have led to a lower threshold, which did not occur. A critical band greater than $10 \mathrm{~Hz}$ would be a surprising finding for this system, given the displacement frequency range over which the system functions.

In contrast to the auditory and tactile sensory systems, the kinesthetic system operates over a much more limited range of input frequencies. Spectral analyses of the temporal profiles of voluntary movements involving the arm and hand indicate that peak frequencies range from 1-8 Hz, depending on the requirements of the activity (Kunesch, Binkofski, \& Freund, 1989). Skilled manual activities performed at high speeds, such as typing or piano playing, usually involve movements in the $4-8 \mathrm{~Hz}$ range (Jones, 1997), whereas exploratory finger movements, such as those involved in tactile recognition tasks, are performed more slowly, with peak frequencies usually being less than $2 \mathrm{~Hz}$ (Kunesch et al., 1989). Activities, such as object manipulation, involving either the hand or an end effector such as a robotic arm, are considered to be highly dependent on sensory feedback and are performed very slowly in order to extract information from the environment.

The bandwidth of voluntary motor responses has also been measured, using a variety of stimuli that subjects are required to track, and the results from these studies generally show maximum bandwidths of between 2 and $5 \mathrm{~Hz}$ (Cathers, O'Dwyer, \& Neilson, 1996; Leist, Freund, \& Cohen, 1987). The signal frequency of $10 \mathrm{~Hz}$ was chosen for the present experiment so that the bandwidth of the noise could span a reasonable range of frequencies around the central frequency. At lower signal frequencies, the problem emerges of producing a noise stimulus with sufficient samples at lower frequencies in a reasonable time interval. 

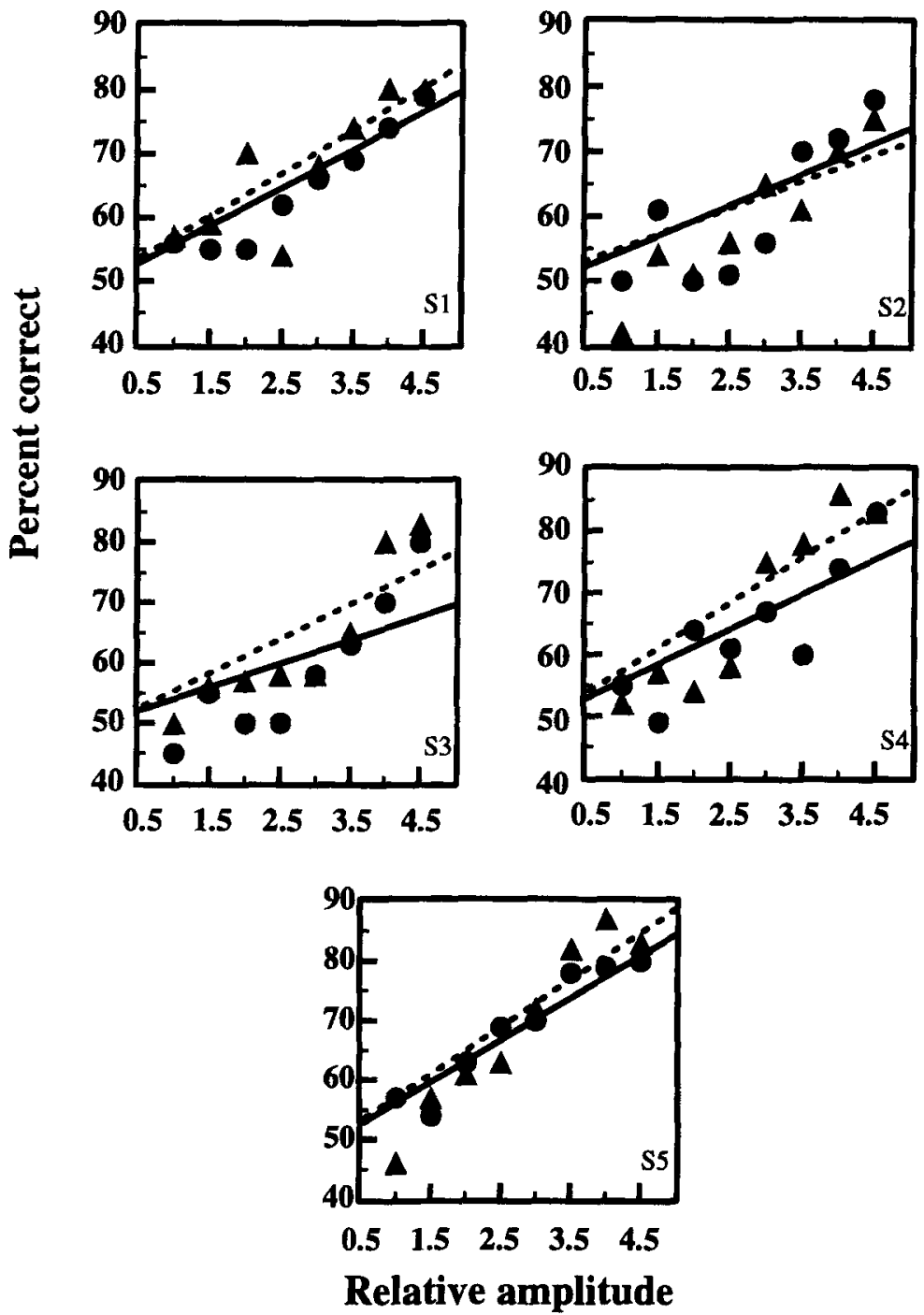

Figure 4. The mean percentage of correct responses at each amplitude for each subject in the two experiments. The circles (solid line) represent the correct responses with a $10-\mathrm{Hz}$ band of noise ( $5-15 \mathrm{~Hz}$ ), and the triangles (dashed line) represent the correct responses with a 4-Hz band of noise $(8-12 \mathrm{~Hz})$. The lines are the least squares regression functions (with one free parameter), fitted to each subject's data.

In the kinesthetic system, information about changes in muscle length and, hence, limb movement is processed by two types of stretch receptor found in muscle, known as primary and secondary spindle receptors. These receptors and their associated neural pathways may be considered to be separate channels, although there is considerable overlap in their responses to changes in muscle length. Both primary and secondary receptors respond to changes in muscle length, but primary spindle receptors are much more sensitive to the velocity component of a lengthening contraction and increase their discharge rates considerably as the velocity of the stretch increases. Secondary spindle receptors show much less dynamic re- sponsiveness and have a more regular discharge rate than primary receptors at a constant muscle length (Prochazka, 1996). The type of stimuli used in the present experiment would be processed by both classes of receptor, although the primary spindle receptors would be more responsive to the variations in amplitude of the higher frequencies included in the stimuli and so may be assumed to be the predominant source of kinesthetic information.

The movement perturbations applied to the arms in the present study not only excited the kinesthetic system but also activated the cutaneous tactile system, as the subjects grasped the cylindrical rods projecting from the motors. The channels that mediate the perception of low- 
frequency vibrotactile stimuli in the skin are the NP I channel, which has a nominal operating range of 10 $100 \mathrm{~Hz}$, and the NP III channel, which operates over a frequency range of $0.4-100 \mathrm{~Hz}$ (Bolanowski et al., 1988). Both of these channels would provide information that could be used to facilitate the subjects' judgments in the present experiments. In order to determine how much the tactile system contributed to the subjects' performance in the present experiments, it would be necessary to eliminate this input, using peripheral anesthesia, and then to measure the thresholds.

In conclusion, it appears that, for the kinesthetic system, the ability to detect a $10-\mathrm{Hz}$ sinusoidal movement stimulus embedded in noise is not influenced by the bandwidth of the noise over a range of $4-10 \mathrm{~Hz}$. This means that the interference resulting from a $10-\mathrm{Hz}$ band is the same as that which occurs with a $4-\mathrm{Hz}$ band. If a critical filter does exist for this system, it would have to be greater than $10 \mathrm{~Hz}$.

\section{REFERENCES}

Bolanowski, S. J., Jr., Gescheider, G. A., Verrillo, R. T., \& CheckOSKY, C. M. (1988). Four channels mediate the mechanical aspects of touch. Journal of the Acoustical Society of America, 84, 1680-1694.

CATHERs, I., O'DWYER, N., \& NeILSON, P. (1996). Tracking performance with sinusoidal and irregular targets under different conditions of peripheral feedback. Experimental Brain Research, 111, 437-446.

Clark, F. J., Burgess, R. C., Chapin, J. W., \& Lipscomb. W. T. (1985). Role of intramuscular receptors in the awareness of limb position. Journal of Neurophysiology, 54, 1529-1540.

FitzPatrick, R., \& McCloskey, D. I. (1994). Proprioceptive, visual and vestibular thresholds for the perception of sway during standing in humans. Journal of Physiology, 478, 173-186.

Fletcher, H. (1940). Auditory patterns. Reviews of Modern Physics, $12,47-65$.

Gandevia, S. C., Hall, L. A., \& McCloskey, D. I. (1983). Proprioceptive sensation at the terminal joint of the middle finger. Journal of Physiology, 335, 507-517.

Gescheider, G. A., Verrillo, R. T., \& Pelli, D. G. (1992). Effects of noise on detection of amplitude increments of sinusoidal vibration of the skin. Journal of the Acoustical Society of America, 91, 348-353.
Gescheider, G. A., Verrillo, R. T., \& Van Doren, C. L. (1982). Prediction of vibrotactile masking functions. Journal of the Acoustical Society of America, 72, 1421-1426.

Goodwin, G. M. (1976). The sense of limb position and movement. Exercise \& Sport Sciences Reviews, 4, 87-124.

HALL, L. A., \& MCCLOSKEY, D. I. (1983). Detections of movements imposed on finger, elbow and shoulder joints. Journal of Physiology, 335, 519-533.

JoNES, L. A. (1997). Dextrous hands: Human, prosthetic and robotic. Presence, 6, 29-56.

Jones, L. A., Hunter, I. W, \& IRwIN, R. J. (1992). Differential thresholds for limb movement measured using adaptive techniques. Perception \& Psychophysics, 52, 529-535.

KoKmen, E., Bossemeyer, R. W., \& Williams, W. J. (1977). Quantitation of motion perception in the digits: A psychophysical study in normal human subjects. Annals of Neurology, 2, 279-284.

Kunesch, E., Binkofski, F., \& FreUnd, H.-J. (1989). Invariant temporal characteristics of manipulative hand movements. Experimental Brain Research, 78, 539-546.

Leist, A., Freund, H.-J., \& Cohen, B. (1987). Comparative characteristics of predictive eye-hand tracking. Human Neurobiology, 6, 19-26.

Makous, J. C., Friedman, R. M., \& Vierck, C. J., JR. (1995). A critical band filter in touch. Journal of Neuroscience, 15, 2808-2818.

MoORe, B. C. J. (1997). An introduction to the psychology of hearing (4th ed.). New York: Academic Press.

Prochazka, A. (1996). Proprioceptive feedback and movement regulation. In L. Rowell \& J. T. Shepherd (Eds.), Handbook of physiology: Section 12. Exercise: Regulation and integration of multiple systems (pp. 89-127). New York: American Physiological Society.

Refshauge, K. M., Chan, R., Taylor, J. L., \& McCloskey, D. I. (1995). Detection of movements imposed on human hip, knee, ankle and toe joints. Journal of Physiology, 488, 231-241.

SCHOONEVELDT, G. P., \& MoORE, B. C. J. (1989). Comodulation masking release (CMR) as a function of masker bandwidth, modulator bandwidth and signal duration. Journal of the Acoustical Society of America, 85, 273-281.

Sheridan, T. B., \& Ferrell, W. R. (1974). Man-machine systems. Cambridge, MA: MIT Press.

TAYLOR, J. L., \& MCCLOSKEY, D. I. (1992). Detection of slow movements imposed at the elbow during active flexion in man. Journal of Physiology, 457, 503-513.

(Manuscript received September 4, 1997; revision accepted for publication February 14, 1998.) 